AperTO - Archivio Istituzionale Open Access dell'Università di Torino

\title{
Hilfsverben und Grammatikalisierung: Die fatale Attraktion von geben
}

\section{This is the author's manuscript}

Original Citation:

Availability:

This version is available http://hdl.handle.net/2318/127963

since

Publisher:

Walter De Gruyter

Published version:

DOI:10.1515/9783110925364.193

Terms of use:

Open Access

Anyone can freely access the full text of works made available as "Open Access". Works made available under a Creative Commons license can be used according to the terms and conditions of said license. Use of all other works requires consent of the right holder (author or publisher) if not exempted from copyright protection by the applicable law. 


\section{Livio Gaeta}

\section{Hilfsverben und Grammatikalisierung: Die fatale Attraktion von geben ${ }^{1}$}

Auxiliaries for the expression of voice have been claimed to grammaticalize from four source categories (Haspelmath 1990). In particular, inactive and causative verbs are said to separately give rise to passive morphology. This paper will be devoted to the grammaticalization of German passive auxiliaries. While the grammaticalization of the fientive verb werden has received much attention, a very interesting instance of passive formation out of causatives usually goes unnoticed: geben as a passive auxiliary in the Middle-Rhine dialects. It goes back to a causative meaning extension observed in the 16th century, which is a fertile seed-bed of future developments. On the one hand, it gave rise to the presentativeexistential construction by way of a conversational implicature, according to which the creation of a new entity implies its existence. A similar conversational implicature explains the development, on the other hand, of a fientive meaning, because the coming into existence of some properties of an entity can be taken to imply that it has acquired such properties. Thereafter, geben spread to the functional space occupied in Standard German by werden. The case of geben shows that different source channels for passive morphology can in fact cross each other.

\section{Passivstrukturen im Deutschen}

Nach allgemeiner Auffassung kommen im Deutschen zwei Arten von Passiv vor, die dadurch unterschieden werden, was für ein Hilfsverb in der Passivperiphrase verwendet wird. Wenn auch unterschiedlich bewertet, weisen die zwei Passivarten eine lange Geschichte auf, die sich nur zum Teil mit der Passiventwicklung in den anderen germanischen Sprachen auf einen gemeinsamen Nenner bringen lässt (siehe Abraham 1989; Eroms 1990). Eines steht jedenfalls fest: Nach allgemeiner Auffassung ist im heutigen Deutsch das werden-Passiv im Gegensatz zum seinPassiv der unmarkierte Passivtyp, wobei „insgesamt werden immer meht Passivaufgaben übernimmt und sein ganz allmählich zurückgedrängt wird“ (Eroms 1992: 238). Außer diesen zwei Passivmöglichkeiten, die die kanonischen Subjekt-Objekt-Rollen betreffen, kommt - besonders in der Umgangssprache - eine weitere

1 Teile dieses Aufsatzes wurden auf der 8th Intemational Cognitize Linguistics Canferenc, Logronho 20.25.07.2003, vorgestellt. Ich bin allen Anwesenden sowie den Herausgebem des vorliegenden Sammelbandes für hilfreiche Kommentare sehr dankbar. 
Passivstruktur vor, die dreistellige Prädikate betrifft, indem das Rezipientenargument zum Subjekt promoviert wird (z.B. Karl bekommt / kriegt / erbält ein Buch von Hanna geschenkl).

Wenn sich auch nicht alle darüber einig sind, wie weit die verschiedenen Passivarten im heutigen Deutsch grammatikalisiert sind, d.h. im weitesten Sinne eine brauchbare grammatische Alternative darstellen, ${ }^{2}$ ist das sich ergebende Bild dennoch ganz klar. Die verschiedenen Prädikate verfügen über grammatische Mittel, um ein Subjekt zu demovieren und ein direktes bzw. indirektes Objekt zu promovieren. Die in dieser Funktion verwendeten grammatischen Mittel bestehen aus einer Periphrase, die ein Partizip II enthält und dazu noch ein Hilfsverb, das für die Passivart entscheidend ist, und zwar aus einer semantischen (Ereignis-bezogenen) oder syntaktischen (Argument-bezogenen) Perspektive. Im folgenden werden zuerst in Abschnitt 2 die verschiedenen Quellen vorgestellt, die zur Entwicklung von Passivmorphologie führen; dann wird in Abschnitt 3 der Fall des luxemburgischen ginn besprochen, und eine Erklärung seiner diachronen Entwicklung vorgeschlagen, die auf Langackers Idee der Subjektivierung beruht. In Abschnitt 4 werden die Schlussfolgerungen gezogen.

\section{Quellen des Passivs in den Sprachen der Welt}

Während die Passivierung mithilfe eines Hilfsverbs in den Sprachen der Welt nicht häufig ist (Haspelmath 1990: 29), ist die Art der lexikalischen Quelle, die an der deutschen Passivbildung mitbeteiligt ist, weit verbreitet. In diesem Zusammenhang erwähnt Haspelmath (ebd.: 39) eine Reihe von Sprachen, die derartige „intransitive inaktive Hilfsverben" zur Passivbildung verwenden. Nach Haspelmath bezeichnet der Terminus, ,inaktiv" keine Stativität, sondern eher NichtAgentivität. Mit anderen Worten: Ein Sachverhalt kann inaktiv, aber dynamisch sein. Dementsprechend sind die Verben sein und sitzen inaktiv und stativ, dagegen werden und fallen inaktiv und dynamisch. Laut Haspelmath ist die Passivmorphologie (wozu auch die Hilfsverben gerechnet werden) auf vier Quellkategorien zurückzuführen: inaktive Hilfsverben, Kausativa, NP-Reflexiva, und generalisierte Subjektkonstruktionen. Die einzelnen Entwicklungsketten, die zur Passivbildung führen, werden in einem Diagramm zusammengefasst, das zeigt, wie das Passiv aus der unabhängigen Konvergenz von aus den verschiedenen Quellen entstehenden Grammemen hervortritt (Haspelmath 1990: 54):

2 Vgl. Leiss (1994: 156): „Der Bereich der Grammatik beginnt mit der W/ahlmöglichkeit von Perspektiven. Grammatik läßt sich als ein Instrumentarium zur Ioslōsung vom faktisch eingenommenen Standpunkt definieren. In der Realität verfügen wir jeweils über nur eine Perspektive. Wir befinden uns entweder innerhalb oder außerhalb von Gegenständen, Sachverhalten oder Situationen. Grammatik liegt dann vor, wenn beide Perspektiven gewählt werden können, unabhängig vom jeweils eingenommenen Standpunkt." 


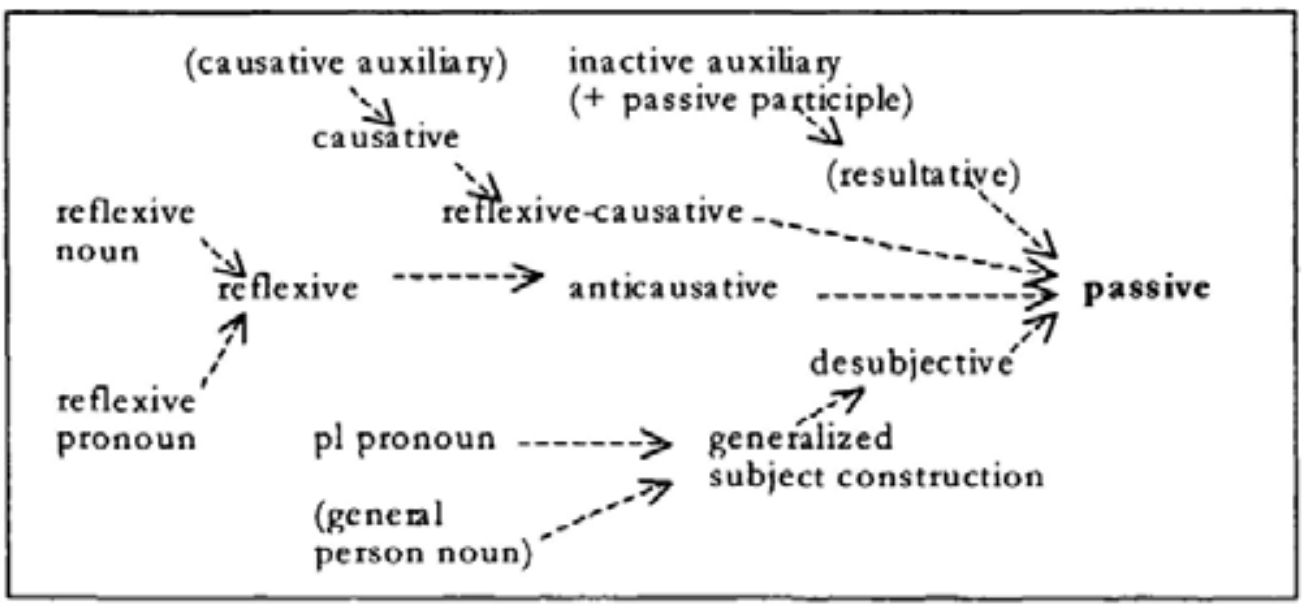

Abb. 1: Passivquellen in den Sprachen der Welt

Genau dem Grammatikalisierungkanal der inaktiven Verben entsprechen die deutschen Hilfsverben werden und sein. Insofern scheint das deutsche Passiv nicht einzigartig zu sein, wenigstens innerhalb der Hilfsverben-geneigten indoeuropäischen Sprachen. Darüber hinaus verwendet das Rezipientenpassiv ein transitives inaktives Verb, das ebenfalls vergleichsweise häufig vorkommt (Haspelmath 1990: 41).

Nach Haspelmath ist der Weg zur Grammatikalisierung des Passivs kohärent und unidirektional: Inaktive Prädikate werden über eine resultative Stufe Passivmarker bzw. Hilfsverben, wie im Deutschen. So weit ist alles klar, und kaum bemerkenswert. Was ist aber zu beobachten, wenn deutsche Mundarten in die Betrachtung einbezogen werden?

\section{Ginn im Luxemburgischen}

Abgesehen von Hilfsverben, die auch in der Standardsprache erscheinen, bieten deutsche Mundarten andere Möglichkeiten an, die mit der kanonischen Passivstruktur verbunden sind. ${ }^{3}$ Insbesondere erscheint in einer kleinen Sprachinsel, die von der Mosel durchquert wird (d.h. im Moselfränkischen, Westpfälzischen, Lothringischen, Schirmunski 1962: 578), das Verb, das etymologisch dem Verb geben entspricht, als Kern der Passivperiphrase. Das Phänomen ist typologisch gesehen ziemlich beschränkt. Eine ähnliche Grammatikalisierungserscheinung ist in

3 Das Verb kommen, das im Bairischen als Passivauxiliar verwendet wird, wird hier außer Acht gelassen. Zum einen ist ein Bewegungsverb als Grammatikalisierungsquelle alles andere als ungewöhnlich (Haspelmath 1990: 38); zum anderen ist der Grammatikalisienungspfad solcher venitiven Verben schon vielfalltig diskutiert worden (u.a. Wiesinger 1989 zum Bairischen; Squartinj 2003 zum Italienischen). 
chinesischen Dialekten festzustellen, wie im folgenden Beispiel (Haspelmath 1990: 48):

(1) Lissì gěi Zhāngsān kànjiàn-le

Lisi geben Zhangsan sehen-PERF

"Lisi wurde von Zhangsan gesehen' (wörtl. ,Lisi gab Zhangsan (ihn) sehen')

Ein solches Beispiel entspricht ganz genau der Passivbildung im Luxemburgischen (Bruch 1973; Bellmann 1998; Nübling 2000: 71-73, Ms.): ${ }^{4}$

(2) ech gi gesinn, ech si gesinn ginn

,ich werde gesehen, ich bin gesehen worden

Es ist das Verb ginn, geben', das die Rolle des Passivauxiliars spielt. Haspelmath (1990: 49) erwähnt die chinesischen Beispiele, wie auch andere Fälle dieser Art in türkischen und tungusischen Sprachen, im Zusammenhang mit den kausativen Quellverben, die zwar weniger verbreitet sind, aber als „well-motivated semantically" erscheinen. Die dahinter stehende semantische Motivation hat damit zu tun, dass in diesem Fall ein Verlust an semantischer Spezifizität, insbesondere Agentivität stattfindet und hierdurch eine passivische Verwendung zustande kommen kann. Insofern fasst Haspelmath alle kausativen Quellverben in einem eigenen Grammatikalisierungskanal zusammen, der dann (über eine reflexivekausative Zwischenstufe) direkt passivische Morphologie ergibt (vgl. Abbildung 1 oben).

Liegt eine ähnliche Erklärung aber auch für das Luxemburgische auf der Hand? Im Luxemburgischen müssen die Verhältnisse komplizierter sein, da ginn nicht nur als Passivauxiliar vorkommt, sondern überhaupt die Funktion des standarddeutschen werden aufweist: ${ }^{5}$

(3) a. ech gi krank, ech gi Schoulmeeschter ,ich werde krank, ich werde Schulmeister'

Kopula

b. wann ech Zäit hätt, géif ech bleiwen würde-Form ,wenn ich Zeit hätte, würde ich bleiben

Synchron ist der Layering-Effekt (d.h. die Heterogrammie, Girnth 2000: 83) auffällig, wenn man die Verwendung von ginn als Vollverb betrachtet:

(4) ech ginn der e Buch

Vollverb

,ich gebe dir ein Buch"

Man beachte, dass ginn als Vollverb im Gegensatz zu seiner Gegenrolle das Hilfsverb haben selegiert: 6

4 Der vorliegende Aufsatz konzentriert sich auf das Luxemburgische. Åhnliche Verhältnisse gelten jedoch auch in anderen Mundarten dieses Sprachraums, z.B. in der Saarbrücker Mundart (Steitz 1981).

5 Die einzige Funktion von werden die dem luxemburgischen ginn fehlt, ist die Verwendung als Futurmarker. Letztere mird im Luxemburgischen präsentisch realisiert (Nübling Ms.: 12).

6 Untersuchungsbedürftig ist, ob die Heterogrammic vollständig ist oder ob noch eine homonymische Beschränkung vorliegt, die die Folge geber(Vollverb) + geber(Hilfsverb) wie in etma e Buch gett der ginblockiert. 
(5) a. ech hunn der eppes ginn

,ich habe dir etwas gegeben

b. ech si krank ginn, ech si gefrogt ginn

,ich bin krank geworden, ich bin gefragt worden

Nun stellen wir eine diachrone Äquivalenz fest: Ginn hat im Luxemburgischen die Funktion von werden übernommen, wobei das Letztere "morphologisch wie semantisch zu den Präteritopräsentia bzw. Modalverben übergegangen [ist] und dem Ausdruck der Vermutung [dient] " (Nübling Ms.: 12).

Eine solche Äquivalenz, d.h. ginn in der Funktion von werden und nicht einfach in der passivischen Funktion, muss als Ausgangspunkt unserer Analyse angesetzt werden. Wie Bellmann (1998), Girnth (2000) und Nübling (Ms.) betonen, ist auch in arealer Hinsicht festzustellen, dass der Sprachraum, in dem geben als passivischer Marker gilt, eine Untermenge des Gebietes ist, in dem es in der Funktion von werden, d.h. als inchoative bzw. fientive Kopula, vorkommt. Erst nach dieser fatalen Attraktion dürfte das fientive geben auch in die Passivperiphrase eingetre-

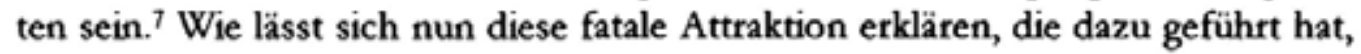
dass sich ein dreistelliges und noch dazu agentivisches Prädikat zu einer inaktiven Kopula entwickelt hat?

\subsection{Missverständnisse im Bereich der geben-Verben}

Bevor wir eine Erklärung dieses verwickelten Sprachwandels versuchen, müssen wir noch ein paar Missverständnisse ausräumen, die in der zitierten Literatur geläufig sind. Nach Bellmann (1998: 252f.) hat die oben beschriebene Entwicklung mit den Haupteigenschaften von geben zu tun, die es mit den anderen Passiv bildenden Hilfsverben teilt. Sie können auf folgende Weise zusammengefasst werden. Erstens handelt es sich um Bewegungsverben im weitesten Sinne, da die Bedeutung von werden im Sinne von Wachstums-Bewegung ohne Ortsveränderung (Di Meola 1994: 28) charakterisiert werden kann; bei geben liegt eine kausative Beförderungs-Bewegung vor. Zweitens sind es ingressive / inchoative Verben, d.h. Verben, die vermöge ihrer Proto-Bedeutung die Veränderung und den Eintritt eines qualitativen Zustandes benennen. Es sind drittens deiktische Verben, wobei die Richtung als solche nicht von Belang zu sein scheint. Die vierte Eigenschaft ist Intransitivität; sie kann im Ursprung aber nur werden (und bair. kommen) zugeschrieben werden.

Obwohl man der Feststellung nur zustimmen kann, dass Kausativität und Telizität in der Grammatikalisierung von geben als Kopula sehr wohl eine Rolle spielen, scheint mir aber das Bild, wie es Bellmann zeichnet, etwas verworren zu sein. Zwar weist Girnth (2000: 144) - Bellmann folgend - darauf hin, dass

7 Es bleibt jedenfalls genauer zu untersuchen, wann und wie geben in diesem Sprachraum dic Stelle von werden übernommen und wieso sich menden darüber hinaus den Modalverben angenähert hat. 
dic den Richtungsverben werden, kommen und geben gemeinsame Origo-Bezogenheit nun genau ihre Verwendung als passivbildende Auxiliare [erklärt]. Aufgrund ihres deiktischen Potentials stützen sie semantisch die Umkehrung der natürlichen Verweisrichtung, die für die Passivkonstruktionen charakteristisch ist.

Aufgrund dieser Erklärung ist aber kaum verständlich, wieso sich ein agentivisches Verb wie geben in ein inaktives Prädikat verwandelt hat, das ein Patiens als Subjekt aufweist. Mit anderen Worten: Unklar bleibt, wann und wie geben einen solchen Intransitivierungsprozess durchlaufen hat. Auch nicht besser wird die Erklärung, wenn man auf die doppelte Funktion von geben hinweist, die es angeblich besonders angemessen macht, um als Hilfsverb in Passivstrukturen verwendet zu werden:

Es verweist qua seiner Origo-Bezogenheit anaphorisch auf das Patienssubjekt und qua seiner spezifischen Besitzwechselperspektive, die auf den alten Besitzer (= Agens) gerichtet ist, kataphorisch auf das im Passivsatz zumeist nicht ausgedrückte Agens (Girnth 2000: 145).

Man beachte, dass diese Erklärung davon ausgeht, dass die Grundsemantik von geben (entsprechend dem Prinzip der semantischen Konstanz, das bei Hopper 1991 dem der Persistenz entspricht) in der passivischen Periphrase beibehalten wird. Dann ist aber nicht klar, wie ein ursprüngliches Agenssubjekt als Patiens umgedeutet werden kann: Schließlich ist es ja eher die Agens-zentrierte OrigoBezogenheit, die mit der Perspektive des Besitzwechsels zu tun hat. Dem Fall von geben kann man deutlich den Gebrauch von bekommen (und von engl. get, Givón / Yang 1994) als passivisches Hilfsverb gegenüberstellen, da im letzten Fall eine Rollenkongruenz zwischen Subjekt und Patiens schon vor der Grammatikalisierung vorliegt. Deshalb ist die Richtung der deiktischen Verben doch von Belang, und es muss klar gemacht werden, wie sich erstens die Kopula-Funktion entwickelt hat, d.h. wie cin ursprüngliches Agenssubjekt inaktiv geworden ist. ${ }^{8}$

\subsection{Kognitive Grundlagen des semantischen Wandels}

Die hier zu klärende Frage wird von Nübling (Ms.: 26) mit einigen zur deutschen Umgangssprache gehörenden Konstruktionen wie es / das gibt ein Gewitter / Regen / eine Katastrophe bzw. mit der Frage was gibt denn das? in Zusammenhang gebracht, aus denen das für den belebten Handlungsrezipienten stehende Dativobjekt verschwunden ist. Meiner Meinung nach ist dieser Hinweis von wesentlicher Relevanz: Der erste Schritt zur Verwandlung von geben in eine Kopula muss in einer natürlichen Bedeutungserweiterung gesucht werden, die für die Geschichte von

8 Es sei nur am Rande darauf hingewiesen, dass die in der Grammatikalisierungsliteratur gewöhnlich zitierten Falle mit geben als Quellwort - abgesehen von dem oben erwähnten chinesischen Beispiel - die Grammatikalisierung von geben als Rezipientenmarker betreffen. Wie Nübling (Ms.: 26) richtig sieht, impliziert dies einen anderen Grammatikalisienungspfad, der nkeine Umkehr, sondern gerade die Beibehaltung der Ausgangsdeixis und die Nutzung der Valenzstellen [enthält]". 
geben weit reichende Konsequenzen gehabt hat. Diese Bedeutungserweiterung stellt keine Eigentümlichkeit des Deutschen dar, sondern ist übereinzelsprachlich häufig. Es handelt sich um eine Erweiterung, die mit der Telizität von geben zu tun hat und zu einem neuen Sachverhalt führt. Newman (1996: 144-147), der diesem Verb aus der Perspektive der kognitiven Linguistik ein ausführliches Buch gewidmet hat, hat diese Bedeutungserweiterung als „emergence / manifestation of entities" bezeichnet. In seinem kognitiv-linguistischen Modell bekommt geben folgende allgemeine Darstellung (Newman 1996: 33-36):

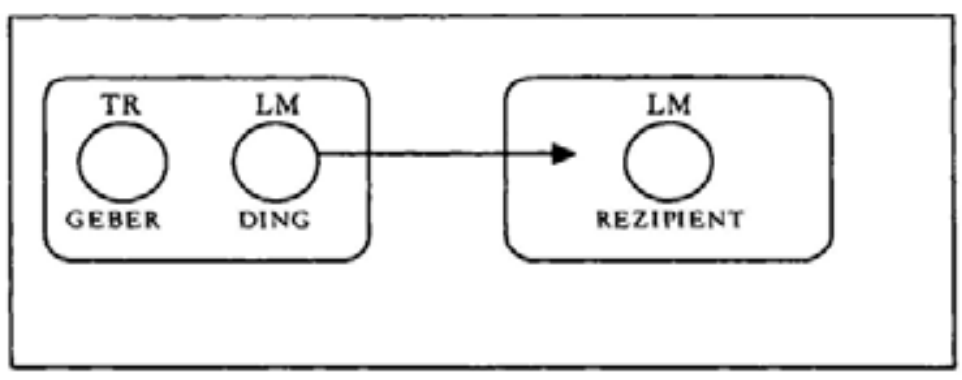

Abb. 2: Grundbedeutung von geben

Ein Geber wird als Trajektor ( $=$ TR) profiliert, hat in seiner Kontrolldomäne ein Ding, das als erste Landmark (= LM) gilt, und verursacht, dass das Ding in eine fremde Kontrolldomäne übergeht, die von einem Rezipienten, der zweiten Landmark, gehalten wird. Das Image-Schema in Abbildung 2 gilt im Allgemeinen für alle wörtlichen Bedeutungen von geben. Für unsere Bedeutungserweiterung ist ein Aspekt dieses Schemas relevant, das mit der Bewegung des Dings außerhalb der Kontrolldomäne des Trajektors zu tun hat. In den Worten Newmans (1996: 144):

The THING may be viewed, therefore, as emerging out of some physical region, and it is this way of viewing the movement of the THING which motivates a large group of extensions involving emergence and manifestation of entities.

Eine solche Bedeutungserweiterung kommt in Beispielen vor, die auf das $\mathrm{Zu}$ standekommen einer gewissen Entität fokussieren wie Der Baum gab viele Früchte. Hier wird das Ergebnis nicht absichtlich vom Subjekt hervorgebracht, d.h. Agentivität wird in Hintergrund gestellt. Darüber hinaus ist an dem Ereignis kein Rezipient mitbeteiligt. Mithilfe eines Image-Schemas können wir diese Bedeutungserweiterung wie folgt darstellen (Newman 1996: 147): 


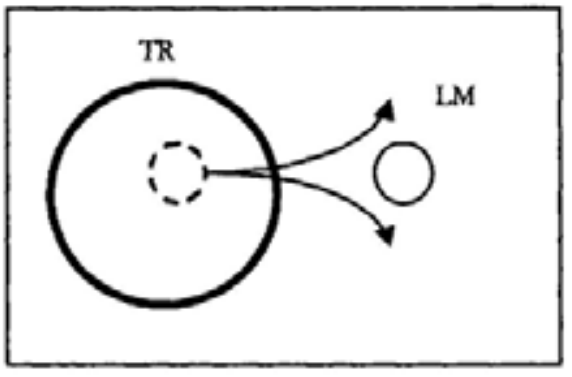

Abb. 3: Bedeutungserweiterung von geben I: Zustandekommen von Entitäten

Aus der Domäne eines Trajektors, der aber im Hintergrund bleibt, tritt eine Landmark heraus, die nur indirekt durch das Agens hervorgebracht wird. ${ }^{9}$ Eine solche Bedeutungserweiterung kann den Bereich der Lebensschöpfung betreffen wie im angegebenen Beispiel, wie auch das Zustandekommen von besonderen (auch abstrakteren) Entitäten; vgl. der Ofen gibt Wärme, der Geiger gibt ein Konzert.

Wenn man weiter von der Domäne des Trajektors abstrahiert, taucht die Bedeutungserweiterung der Offenbarung einer Entität auf, die genau wie das Zustandekommen aus einer Domäne aussieht, mit dem relevanten Unterschied, dass die Quelldomäne nicht mehr profiliert ist (Newman 1996: 157):

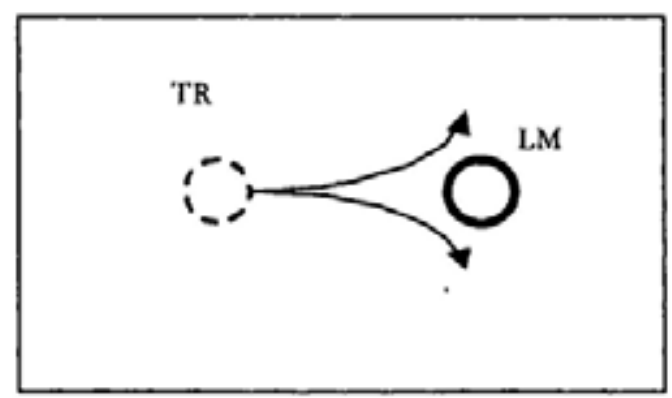

Abb. 4: Bedeutungserweiterung von geben II: Hintergrundstellung des Trajektors

In diesem Image-Schema wird das Agens endgültig in den Hintergrund gerückt. Die zustandekommende Entität wird so wahrgenommen, als ob sie aus einer unbestimmten Quelle käme (in Abbildung 4 mit einer gestrichelten Linie dargestellt). Die ursprüngliche Kausitivität von geben erscheint in dieser Bedeutungserweiterung als vollkommen ausgelöscht; „GIVE verbs used to convey this sense

9 Newman (1996:146) bemerkt hierzu noch: „[A]lthough the schematic emergent sense [...] does not require any human instigation, particular instantiations of the schema may involve humans as the trajectors, [...] and these humans may exert varying degrees of control over the emergence of the landmark entities." Vgl. Ausdrücke wie keinen Lawt / Ton won sich geben, Unsinn ton sich geben, usw. 
tend to be used in constructions which lack the typical agent-patient contrast" (Newman 1996: 156). Trotz der Bedeutungserweiterung nicht ausgelöscht wird dagegen die Telizität, d.h. die ursprüngliche Besitzwechselperspektive, die hier als Offenbarung einer früher nicht bestehenden Entität erscheint und demzufolge als Inchoativität bzw. Resultativität uminterpretiert werden kann. Daher gilt: „The construction types favoured to convey the notion of manifestation of an entity are reflexive, impersonal, passive, and intransitive" (Newman 1996: 156).

Was die reflexive Entwicklung von geben angeht, kann hier nur kurz auf die sog. präsentativen Konstruktionen hingewiesen werden, für die gilt: „[T]he sense of emergence out of a separate entity is transformed into some process involving just one entity in the real world" (Newman 1996: 158). Im folgenden werden drei Beispiele genannt, die dieser reflexiven Verwendung entsprechen und drei unterschiedliche Perspektiven belegen:

(6) a. Es begab sich, dass ...

b. Es wird sich schon geben.

c. Sie gab sich ganz natürlich.

In (6)a. ist der einfachste Fall dargestellt; in (6)b. ist die präsentative Konstruktion mit der Implikation verbunden, dass sich der Sachverhalt positiv bzw. den Erwartungen angemessen entwickeln wird; in (6)c. ist es das belebte Subjekt der präsentativen Konstruktion, das in einer bestimmten Weise auftritt.

Mit der unpersönlichen Konstruktion ${ }^{10}$ kommen wir zu einer entscheidenden Bedeutungserweiterung, die nicht nur große Konsequenzen für das Deutsche mit sich bringt, sondem auch relevante Hinweise zum Begreifen der Entwicklung von geben zur Kopula liefert. Deswegen werden wir nun auch die diachrone Entwicklung näher betrachten, wie sie sich aus der detaillierten Rekonstruktion von Newman (1998) ergibt. Im Standarddeutschen ist die unpersönliche Konstruktion als (präsentativ-)existenziell aufzufassen, wobei sie entweder auf die Anwesenheit von gewissen Entitäten in einem gegebenen Raum oder auf die schlichte Existenz hinweist:

(7) a. Es gibt viele Kinder in der Schule.

b. Es gibt einen Gott.

Wie sich eine solche unpersönliche existenzielle Konstruktion entwickelt hat, kann man mithilfe des Offenbarungsschemas in Abbildung 4 begreifen, das eine direkte Abstraktion vom Image-Schema in Abbildung 3 darstellt. Der Abstraktionsprozess, der zu dieser unpersönlichen Konstruktion geführt hat, ist schon vor langer Zeit rekonstruiert worden und hat unmittelbar mit dem Zustande-

10 Es sei hier nur am Rande auf die passivische Verwendung von geben hingewiesen, die Newman (1996: 166) so beschreibt: ${ }_{n} \mathrm{~A}$ passive construction with the agent unspecified achieves the effect of highlighting one entity in a scene or event". Vgl. schwed. Det gite-s (wörtl. gibl-PASs) losming "Es gibt eine Lösungt und deutsche Konstruktionen wie gegeben dass ... . Beachte femer auch dic intransitive Verwendung von geben wie in dem englischen Ausdruck What gires? ,Was passiert?" (ebd.:168). 
kommen von Entiäten zu tun: das Wetter ist sebr gïnstig: das - es gibt gute Ernte (Grimm 1837: 266; Behaghel 1924: 137). Diese Konstruktion erscheint selten in mittelhochdeutscher Zeit, wird aber erst im Frühneuhochdeutschen wesentlich häufiger (Kehrein 1856: 56). Besonders häufig erscheint sie bei dem Straßburger Schriftsteller Fischart, auf dessen Werken Newmans (1998) Untersuchung basiert. Typischerweise erscheint bei Fischart die es gibt-Konstruktion als einer mehr oder weniger expliziten Voraussetzung unterworfen wie in den folgenden Beispielen aus der Geschichtklitterung (1575):

(8) a. wann nur alte Weiber unnd die Hund dran seychten, so gebs guten Burgundischen Saltpeter (Fischart: Geschichtelitterung, 125, 37-38)

b. sonst wo die Erd sich zwischen Sonn und Mon einlegt, so gibts finsternuß (Fischart: Geschichtklitterung, 99, 13-14)

c. O wic ernsthaft betten gibt es alsdann für ihn, daß er wider gesund heimkomme (Fischart: Geschichtklitterung, 103, 4-5)

d. Dann es gibt gestolene Kind ... (Fischart: Geschichtklitterung, 88, 6-7)

Die ersten drei Beispiele belegen die bei Fischart meist vorkommenden es gibtStrukturen, in denen "there is a dynamic component in the meaning of es gibt, associated with the development, emergence, or creation of an entity " Newman 1998: 313). In (8)a.-b. liegt eine materielle Vorbedingung vor, auf die durch es direkt verwiesen wird und die dazu führt, dass eine konkrete Entität (der W ein, vgl. (8)a.) bzw. eine abstrakte Entiät (die Finsternis, vgl. (8)b.) zustande kommt. In (8)c. ist diese Vorbedingung nicht direkt vorhanden; sie erscheint aber zuvor im Kontext und hat mit der Abfahrt des Bräutigams zu tun, was als Antezedens mitverstanden werden kann. Der Fall in (8)d. entspricht ziemlich genau der heutigen existenziellen Verwendung, ist aber seltener zu finden. Aufgrund dieser Antezedens-Konsequenz-Beziehung hat sich dann mithilfe einer konversationellen Implikatur die existenzielle Bedeutung entwickelt (Newman 1998: 321):

Tab. 1: Implikaturenreihe I: Existentielle Bedeutung

\begin{tabular}{|c|c|c|}
\hline \multirow[t]{2}{*}{$A$} & $X_{\text {gibt }} Y$ & $\begin{array}{l}\text { Es liegt eine kausale Beziehung zwischen einer Entität } \mathrm{X} \text { und dem Zu- } \\
\text { standekommen einer anderen Entität } \mathrm{Y} \text { vor. }\end{array}$ \\
\hline & & $\downarrow$ \\
\hline B & Es gibt $Y$ & Infolgc eincs bestimmten Antezedens kommt eine Entität Y zustande. \\
\hline \multirow[t]{2}{*}{$\mathrm{C}$} & Es gibt $Y$ & $\begin{array}{l}\text { Infolge unbestimmter vorhergehender Ereignisse kommt eine Entität } \mathrm{Y} \text { zu- } \\
\text { stande. }\end{array}$ \\
\hline & & $\downarrow$ \\
\hline $\mathrm{D}$ & Es gibt $Y$ & Y liegt vor. \\
\hline
\end{tabular}

Solche konversationellen Implikaturen sind, wie die Forschung in letzter Zeit erwiesen hat, insbesondere im Bereich der Grammatikalisierung extrem fruchtbar 
(Traugott 1995, 1999; Detges 2000; Girnth 2000: 75-81). Aufgrund der konversationellen Implikaturen kam es zu einer abstrakteren Bedeutungserweiterung, die zur grammatikalisierten es gibt-Struktur geführt hat. Entscheidend für die semantische Entwicklung war die Abwesenheit bzw. das Verschwinden eines kausalen Antezedens, das mit dem Eintreten eines neuen Sachverhaltes in Zusammenhang gebracht werden konnte.

Parallel zu dieser Bedeutungserweiterung verläuft eine ähnliche semantische Entwicklung, die von demselben Image-Schema in Abbildung 3 ausgeht. Diesmal ist aber eine andere konversationelle Implikatur von Belang, die direkt mit der Erzeugungs-Bedeutung zu tun hat. Wie oben angedeutet, ist diese Bedeutungserweiterung

widely attested in languages (it is extensive, for example, in the Romance languages), and motivations for this semantic shift can be found in the conceptual similarities between, give' and ,produce, yield'. Thus, just as, give' involves the movement of a thing from out of the sphere of control or possession of the giver, so ,produce, yield' involves a kind of movement of a new entity out of a physical region associated with the producing entity (Newman 1998: 317).

Eine solche Bedeutungserweiterung kann man in folgenden frühneuhochdeutschen Beispielen aus dem 16. Jahrhundert feststellen:

(9) a. spannen sie tücher oben in auf die dächer, dasz sie in der mitte herab hangen und einen sack geben (DWB, Stw. geben)

b. jeder wil bei diesem kinde willig einen paten geben (DWB, Stw. geben)

In diesen Beispielen ist die herauskommende Entität durch einen zunehmenden Abstraktionsgrad charakterisiert und gleichzeitig mit der „produzierenden Entität" assoziiert. Wenn nun in dieser Bedeutungserweiterung eine neue Entität zustande kommt, die eine natürliche Entwicklung bzw. Erweiterung der Quelle darstellt, entstehen die Voraussetzungen für eine konversationelle Implikatur. Man beachte die folgenden Beispiele, die erneut aus Fischarts Geschichtklitterung stammen und von Newman (1998: 316) im Zusammenhang mit der es gibt-Konstruktion erwähnt werden:

(10) a. als vil all Berge Trauben geben (Fischart: Geschichtklitterung, 82, 22-23)

b. verzicht mir, daß ich euch den Säuen vergleich, sie geben dannoch guten Speck (Fischart: Geschichtklitterung, 56, 30-31)

Während in (10)a. die Weintrauben nur als Produkt der Berge aufgefasst werden können, stellt Speck in (10)b. eine natürliche Entwicklung eines Schweins (und sogar einen Teil des Tieres selbst) dar. Daraus kann die Implikatur entstehen: Falls X die Entität / Eigenschaft Y entwickelt, dann wird X zu Y. Diese Erweiterung liegt folgenden Beispielen zugrunde:

(11) a. gebst ein guten Goldschmicd (Fischart: Geschichtklitterung, 123, 25)

b. Geltet ihr Fronecken, welche nit gem spinnen, die geben gute Wirtin? (Fischart: Geschichtklitterang, 135, 29-30) 
Wie Newman (1996: 169) vermutet, erscheint diese Erweiterung "close to the extensions [...] associated with biological change, where GIVE is used to conceptualize processes such as the reproduction and growth of living things". In jenen Fällen handelt es sich um die organische Entwicklung einer Entität, in diesem Fall handelt es sich eher um einen Wandel der Eigenschaften einer Person, der aber nicht organisch bedingt ist. In dieser Perspektive gilt: „[B]ecoming may be construed as a type of emergence of a new form of a thing, but not restricted to organic growth“ (ebd.). Diese Bedeutungserweiterung kann nun als die Abfolge der folgenden Implikaturen zusammengefasst werden:

Tab. 2: Implikaturenreihe II: Inchoative Bedeutung

A $X$ gibt $Y$ Es liegt eine kausale Beziehung zwischen einer Entiät $\mathrm{X}$ und dem $\mathrm{Zu}$ standekommen einer anderen Entität $\mathrm{Y}$ vor.

B $X$ gibt $Y$ Es liegt eine kausale Beziehung zwischen einer Entität $\mathrm{X}$ und dem $\mathrm{Zu}$ standekommen einer anderen Entität $\mathrm{Y}$ vor, die eine natürliche Erweiterung von $\mathrm{X}$ darstellt.

$\downarrow$

C $X$ gibt $Y_{x}$ Eine neue Entität / Eigenschaft $Y_{\mathrm{x}}$ kommt zustande, die als Entwicklung von Eigenschaften begriffen wird, die $\mathrm{X}$ eigen sind.

D $X$ gibt $Y_{x} \quad \mathrm{X}$ wird $\mathrm{Y}_{\mathbf{x}}$.

Der entscheidende Wendepunkt besteht in der Konzeptualisierung der neu zustande kommenden Entität als natürliche Entwicklung von X. Somit entsteht eine konzeptuelle Kontinuität bzw. Identität zwischen $\mathrm{X}$ und ihrer Erweiterung $\mathrm{Y}_{\mathrm{x}} \cdot{ }^{11}$

Der Hinweis auf Fischarts Werke mag zufällig erscheinen. Die hier vorgestellte Rekonstruktion zeigt aber, dass die frühe Überlieferung der es gibt-Konstruktion mit der Entwicklung von geben als Kopula in Zusammenhang steht. Man beachte die Fruchtbarkeit von geben im betreffenden Sprachgebiet: Hier finden sich die ersten festen Belege der existenziellen Konstruktion und hier liegen die Voraussetzugen für die Entwicklung als Kopula vor. Es ist gezeigt worden, dass die beiden Entwicklungen auf der konzeptuellen Ebene ziemlich nahe beieinander liegen. Diese Entwicklungen lassen sich auf einen einheitlichen Sprachraum beziehen, der grundsätzlich das Westmitteldeutsche darstellt und entlang des

11 Der weitere Wandel von geben in eine Kopula mag sicherlich auch dadurch begünstigt worden sein, dass in diesem Sprachraum massive Ausgleiche der Kasusendungen, besonders zwischen Nominativ und Akkusativ, stattfanden (Schirmunski 1962: 465-467). Die Reanalyse des zweiten Arguments als nominativisch markiert lag dadurch auf der Hand. Austausche zwischen Nominativ und Akkusativ sind jedenfalls auch bei der es gibt-Konstruktion geläufig (vgl. das Straßburger Beispiel aus DWB, Stw. geben: so herei gitts in der stadt kein junger burst). 
Rheins verläuft. Die Vermutung liegt nahe, dass geben genau in diesem Gebiet die hier skizzierten Grammatikalisierungsprozesse erstmals durchlaufen hat. Die Standardsprache haben sie nur zum Teil, und vergleichsweise später, erreicht.

\subsection{Die fientive Bedeutungserweiterung als Subjektivierung}

Als Ergebnis der oben skizzierten konversationellen Implikaturen kann nun der Sprachwandel als Subjektivierung erfasst werden. Laut Langacker (1999: 297) ist Subjektivierung ,a shift from a relatively objective construal of some entity to a more subjective one“. Im Normalfall wird eine Beziehung XY als objektiv erfasst, wenn sie im Fokus der Dialektik Sprecher-Hörer steht (die als conceptualizers C erscheinen; Langacker 1991: 325):

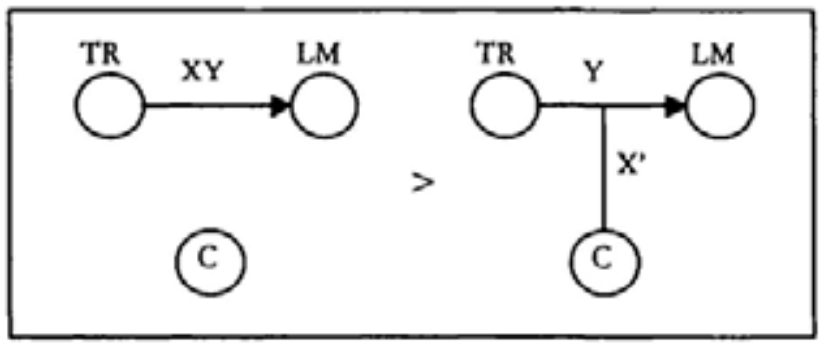

Abb. 5: Allgemeiner Prozess der Subjektivienung

Subjektivierung kann man als "the realignment of some relationship from the objective axis to the subjective axis" (Langacker 1991: 326) auffassen. In Abbildung 5 wird X, d.h. eine Komponente der Beziehung XY, als subjektiv und demzufolge als neue Beziehung $\mathrm{X}^{\prime}$ uminterpretiert, die nun direkt im Zusammenhang mit einigen Aspekten von $C$ begriffen wird. Typischerweise ist Subjektivierung mit Fällen von Verminderung (engl. attenuation) des Kontrollgrades eines agentivischen Subjekts verbunden, und daher taucht eine solche Verminderung in vielen Fällen von Grammatikalisierung auf (Langacker 1999: 306-308). Versuchen wir nun zu verstehen, wie sich diese Idee der Subjektivierung mit der Grammatikalisierung von geben als Kopula im Westmitteldeutschen in Zusammenhang bringen lässt.

Im folgenden Bild wird die Rolle des externen Wahrnehmers (= C) dargestellt, der das geben-Ereignis mit seinen Augen erfasst:

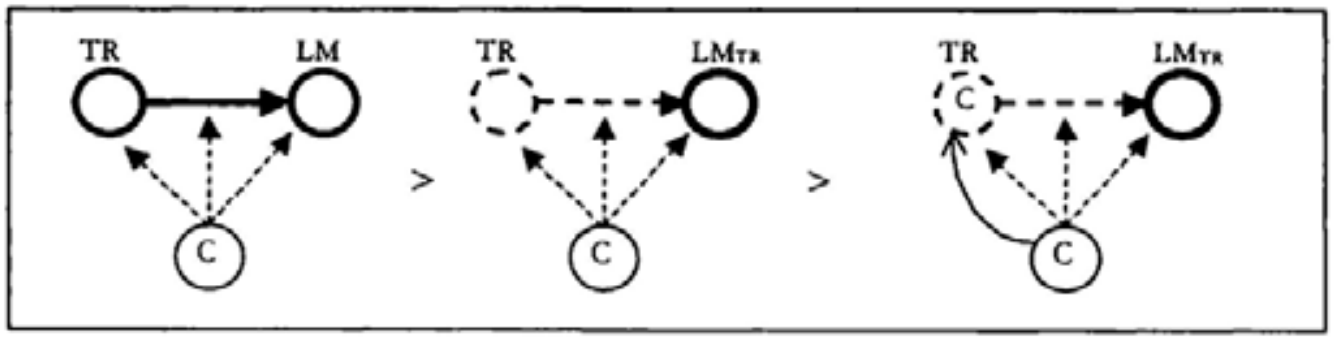

Abb. 6: Die Rolle des Wahmehmers in der Subjektivierung 
Im linken Image-Schema wird das Ereignis objektiv wahrgenommen: Das $\mathrm{Zu}$ standekommen einer Entität wird im Zusammenhang mit der Tätigkeit eines Trajektors dargestellt. ${ }^{12}$ Im darauf folgenden Image-Schema erscheint die Tätigkeit des Trajektors als vermindert, und zwar in Bezug sowohl auf die objektiv aufgefasste Beziehung als auch auf die Rolle, die der Trajektor darin spielt:

Although the conceptualizer continues to carry out the same or a comparable mental scanning as in the initial configuration, the objective situation now offers less motivation for it (Langacker 1999: 299).

Das ursprüngliche Ereignis, das als solches (d.h. „objektiv“) von C wahrgenommen wurde, wird infolge der Subjektivierung in Abwesenheit jeder objektiven Basis für die mentale Tätigkeit von $\mathrm{C}$ uminterpretiert. Diese Uminterpretierung fand aufgrund der in Tabelle 2 gezeigten Reihe von konversationellen Implikaturen statt. Demzufolge gilt: „A relationship is still established between the trajector and landmark, but the basis for it now resides exclusively in the conceptualizer's activity" (Langacker 1999: 299). Letzteres wird im dritten Image-Schema so dargestellt, dass sogar $\mathrm{C}$ als Subjektrolle in das subjektiv konzeptualisierte Ereignis mit einbezogen werden kann, wie etwa in den Beispielen in (2) oben. Die Beziehung ist aber subjektiv konstituiert, d.h. , inheres in the process of conceptualization itself, rather than being an onstage object of conception" (ebd.). Aus dieser Perspektive kann der Subjektivierungsprozess nun als eine Art Teleskopierung der in Tabelle 2 gezeigten Reihe von Implikaturen aufgefasst werden, da wie im phonologischen Wandel „collapsing of two rules which are in a feeding relation to a single rule" (Dressler 1985: 62) nach dem Schema $\mathrm{A} \rightarrow \mathrm{B} \rightarrow \mathrm{C}>\mathrm{A} \rightarrow \mathrm{C}$ stattfindet. Das ist z.B. der Fall im Bretonischen, wo die kontextgebundene Regel $d \rightarrow \delta$ (zwischen Sonoranten) und die kontextfreie Regel $\delta \rightarrow z$ in eine einzige Regel $d \rightarrow z$ (zwischen Sonoranten) zusammengefallen sind. Im Allgemeinen führt Teleskopierung zu einer Erhöhung des Opazitätsgrades der phonologischen Alternationen. Ähnlich veranlasste die Teleskopierung der Implikaturenreihenfolge den Verlust an Transparenz der (diskursbezogenen) semantischen Erweiterung und dementsprechend eine Zunahme an sprecherbezogenem Interpretationsbedürfnis, d.h. eine Subjektivierung.

\section{Fazit}

Die Untersuchung des luxemburgischen ginn hat sich aus einer Reihe von Gründen als fruchtbar erwiesen. Zum einen stellt dieser Passivtyp in Europa eine Rarität dar, die daher zur vollständigeren Erfassung der in diesem Sprachraum beleg-

12 V $V_{\mathrm{gl}}$. Langacker (1999: 298): „[T]he conceptualizer, C, does some kind of mental scanning from the the tmjector to the landmark in concciving of the profiled relationship (i.e. the trajector functions as a ,starting point' or initial point of access in conceptualizing it) ${ }^{a}$. 
ten Passivtypen beiträgt. Zum anderen ist dieser Passivtyp insofern interessant, als er Haspelmaths allgemeines Bild der Entstehungsquellen für die Passivmorphologie wesentlich modifiziert bzw. bereichert. In der obigen Abbildung 1 erscheinen die zur Passivbildung führenden Pfade als von den Quellkategorien der inaktiven und kausativen Verben vollkommen getrennt. Wie aber das luxemburgische ginn deutlich zeigt, brauchen die verschiedenen Grammatikalisierungskanäle nicht so streng voneinander getrennt zu sein, wie Haspelmath vermutet. Tatsächlich war für geben das Auftauchen einer (zunächst kausativen, dann) fientiven Bedeutung der Impuls für seine künftige Entwicklung, wobei geben auf das Gleis von werden geriet und somit ein ähnliches Schicksal teilen konnte. Insofern ist der geben-Fall hochbedeutsam: Das Sichüberkreuzen der verschiedenen Quellkanäle der Passivbildung scheint im Gegensatz zu der allgemeinen Abbildung 1 durchaus möglich zu sein; in diesem Fall muss dann eine direkte Entwicklung vom Kausativum zur inaktiven Kopula angenommen werden.

Darüber hinaus muss für jeden Sprachwandel genau untersucht werden, welche Rolle in der gesamten Entwicklung des Diathesesystems mögliche layering Effekte spielen. Die ursprüngliche kausative Bedeutungserweiterung von geben erwies sich als eine fruchtbare Quelle für künftige Entwicklungen. Einerseits entwickelte sich das kausative geben aufgrund einer konversationellen Implikatur, derzufolge das Zustandekommen von neuen Entitäten ihre Existenz voraussetzt, in die Richtung der existenziellen es gibt-Konstruktion. Andererseits erklärt eine ähnliche Implikatur die Entwicklung einer fientiven Bedeutung, die im Bereich des das geben-Passiv aufweisenden Sprachraums vorkommt. Nachdem geben eine solche fientive Bedeutung erworben hat, kreuzt sein Schicksal das schon weit grammatikalisierte Hilfsverb werden und erfährt eine ähnliche Entwicklung. Schließlich erweist sich diese scheinbar merkwürdige Konstruktion, wenigstens aus historischer Perspektive, als konzeptuell nicht sehr weit von der standarddeutschen Existenzkonstruktion entfernt.

\section{Literatur}

Abraham, Werner (1989): „Zu den distributionellen Eigenschaften von wairdhan ,werden' und wisan ,sein' im gotischen Passiv." In: Walter Tauber ( $\mathrm{Hg}$.), Aspekte der Germanistik. Festschrift für Hans-Friedrich Rosenfeld zum 90. Geburtstag. (Göppinger Arbeiten zur Germanistik 521.) Göppingen: Kümmerle, 601-620

Behaghel, Otto (1924): Deutsche Syntax. Eine geschichtliche Darstellung. Bd. II: Die Wortelassen und Wortformen. B. Adverbium. C. Verbum. (Germanische Bibliothek 1.1.10.2.) Heidelberg: Winter 
Bellmann, Günter (1998): „Zur Passivperiphrase im Deutschen. Grammatikalisierung und Diskontinuität.“ In: Peter Ernst / Franz Patocka (Hg.), Deutsche Sprache in Raum und Zeit. Festschrift fuir Peter Wiesinger zum 60. Geburtstag. Wien: Edition Präsens, 241-269

Bruch, Robert (1973): Luxemburger Grammatik in volkstümlichem Abriß. 3. Auflage. (Beiträge zur Luxemburgischen Sprach- und Volkskunde 10.) Luxemburg: Editions de la Section de Linguistique de l'Institut grand-ducal

Detges, Ulrich (2000): „Time and Truth: The Grammaticalization of Resultatives and Perfects within a Theory of Subjectification." In: Studies in Language 24, 345-377

Di Meola, Claudio (1994): Kommen und gehen. Eine kognitiv-linguistische Untersuchung der Polysemie deiktischer Bewegungsverben. (LA 325.) Tübingen: Niemeyer

Dressler, Wolfgang Ulrich (1985): Morphonology. The Dynamics of Derivation. (Linguistica extranea 12.) Ann Arbor: Karoma

DWB (1878): Deutsches Wörerbuch. Von Jacob und Wilhelm Grimm. Vierten Bandes erste Abtheilung. Erste Hälfte. Forschel-Gefolgsmann. Leipzig: Hirzel

Eroms, Hans-Werner (1990): „Zur Entwicklung der Passivperiphrase im Deutschen." In: Anne Betten (Hg.), Neuere Forschungen zur historischen Syntax des Deutschen. Referate der internationalen Fachleonferenz Eichstätt 1989. (RGL 103.) Tübingen: Niemeyer, 82-97

Eroms, Hans-Werner (1992): „Das deutsche Passiv in historischer Sicht." In: Ludger Hoffmann (Hg.), Deutsche Syntax. Ansicbten und Aussichten. Jahrbuch 1991 des IdS.) Berlin / New York: de Gruyter, 225-249

Fischart, Johann (1963): Gescbicbtelitterung (Gargantua). Text der Ausgabe letzter Hand von 1590. Mit einem Glossar herausgegeben von Ute Nyssen. Düsseldorf: Rauch

Girnth, Heiko (2000): Untersuchungen zur Theorie der Grammatikalisierung am Beispiel des Westmitteldeutschen. (RGL 223.) Tübingen: Niemeyer

Givón, Talmy / Yang, Lynne (1994): „The Rise of the English get-Passive“. In: Barbara A. Fox / Paul J. Hopper (Hg.), Voice. Form und Function. (TSL 27.) Amsterdam / Philadelphia: Benjamins, 119-149

Grimm, Jacob (1837): Deutsche Grammatik. Vierter Theil. Erste Hälfte. Göttingen: Dieterich

Haspelmath, Martin (1990): „The Grammaticization of Passive Morphology. “ In: Studies in Language 14, 25-72

Hopper, Paul J. (1991): „On Some Principles of Grammaticization.“ In Elizabeth C. Traugott / Bernd Heine ( $\mathrm{Hg}$.), Approaches to Grammaticalization. Bd. I: Focus on Theoretical and Methododological Isswes. (TSL 19.1.) Amsterdam / Philadelphia: Benjamins, 17-35

Kehrein, Joseph (1856): Grammatik der deutschen Sprache des funfzehnten bis siebenzebnten Jabrhunderts. Dritter Theil: Syntax des einfachen und mebrfachen Satzes. Leipzig: Wigand 
Langacker, Ronald W. (1991): Concept, Image, and Symbol. The Cognitive Basis of Grammar. (Cognitive Linguistics Research 1.) Berlin / New York: Mouton de Gruyter

Langacker, Ronald W. (1999): Grammar and Conceptualization. (Cognitive Linguistics Research 14.) Berlin / New York: Mouton de Gruyter

Leiss, Elisabeth (1994): „Markiertheitszunahme als natürliches Prinzip grammatischer Organisation (am Beispiel der Verbalkategorien Aspekt, Tempus und Modus). " In: Klaus-Michael Köpcke (Hg.), Funktionale Untersuchungen zur deutschen Nominal- und Verbalmorphologie. (LA 319.) Tübingen: Niemeyer, 149-160

Newman, John (1996): Give: A Cognitive Linguistic Study. (Cognitive Linguistics Research 7.) Berlin / New York: Mouton de Gruyter

Newman, John (1998): „The Origin of the German es gibt Construction." In: ders. (Hg.), The Linguistics of Giving. (TSL 36.) Amsterdam / Philadelphia: Benjamins, 307-325

Nübling, Damaris (2000): Prinzipien der Imegularisierung. Eine kontrastive Analyse von zehn Verben in zehn germanischen Sprachen. (LA 415.) Tübingen: Niemeyer

Nübling, Damaris (Ms.): „Auf Umwegen zum Passivauxiliar - Die Grammatikalisierung von geben, werden, kommen und bleiben im Luxemburgischen, Deutschen und Schwedischen. “ Unveröff. Ms., Universität Mainz 2003

Schirmunski, Victor M. (1962): Deutsche Mundartkunde. Vergleichende Laut- und Formenlehre der dewtschen Mundarten. (Veröffentlichungen des Instituts für deutsche Sprache und Literatur 25.) Berlin: Akademie Verlag

Squartini, Mario (2003): „La grammaticalizzazione di < venire + participio> in italiano: anticausativo o risultativo?" In: Claus D. Pusch / Andreas Wesch (Hg.), $V$ erbalperiphrasen in den (ibero-)romanischen Sprachen. Hamburg: Buske, 23-34

Steitz, Lothar (1981): Grammatik der Saarbrücker Mundart. (Beiträge zur Sprache im Saarland 2.) Saarbrücken: Saarbrücker Druckerei und Verlag

Traugott, Elizabeth C. (1995): „Subjectification in Grammaticalization." In: Dieter Stein / Susan Wright (Hg.), Subjectivity and Subjectivisation. Linguistic Perspectives. Cambridge: Cambridge University Press, 31-54

Traugott, Elizabeth C. (1999): "The Rhetoric of Counter-Expectation in Semantic Change: A Study in Subjectification." In: Andreas Blank / Peter Koch (Hg.), Historical Semantics and Cognition. (Cognitive Linguistics Research 13.) Berlin / New York: Mouton de Gruyter, 177-196

Wiesinger, Peter (1989): „Zur Passivbildung mit kommen im Bairischen.“ In: Wolfgang Putschke / Werner Veith / Peter Wiesinger ( $\mathrm{Hg}$.), Dialektgeographie und Dialektologie. Günter Bellmann zum 60. Geburtstag von seinen Schülern und Freunden. (Deutsche Dialektgeographie 90.) Marburg: Elwert, 256-268 
Brought to you by | Universita Studi di Torino Biblioteca Ruffini (Interscientia S.A.S.)

Authenticated | 172.16.1.226

Download Date | 4/2/12 7:18 PM 